\title{
Deformation of big pseudoholomorphic disks and application to the Hanh pseudonorm
}

\author{
BORIS KRUGLIKOV \\ Mat-Stat. Dept., University of Troms $\varnothing$, Norway \\ kruglikov@math.uit.no
}

\begin{abstract}
We simplify proof of the theorem that close to any pseudoholomorphic disk there passes a pseudoholomorphic disk of arbitrary close size with any pre-described sufficiently close direction. We apply these results to the Kobayashi and Hanh pseudodistances. It is shown they coincide in dimensions higher than four. The result is new even in the complex case.
\end{abstract}

We aim here to prove the following statement, which was proved by another (analogous to the approach of [NW]) and more complicated method in [K1].

Theorem 1. Let $\left(M^{2 n}, J\right)$ be an almost complex manifold and

$$
f_{0}:\left(D_{R}, i\right) \rightarrow(M, J), \quad\left(f_{0}\right)_{*}(0) e=v_{0} \neq 0,
$$

be a pseudoholomorphic disk. Here $e=1$ is the unit vector at $0 \in \mathbb{C}$. For every $\varepsilon>0$ there exists a neighborhood $\mathcal{V}_{\varepsilon}\left(v_{0}\right)$ of the vector $v_{0} \in T M$ such that for each $v \in \mathcal{V}_{\varepsilon}$ there is a bit smaller pseudoholomorphic disk

$$
f:\left(D_{R-\varepsilon}, i\right) \rightarrow(M, J), \quad f_{*}(0) e=v .
$$

The approximating curve $f$ can be embedded/immersed if such is the curve $f_{0}$.

This theorem was used in [K1] for the proof of equivalence of two definitions of Kobayashi pseudodistance $d_{M}$ in almost complex category. In the second $d_{M}$ is associated via path integration to the Kobayashi-Royden pseudonorm:

$$
F_{M}(v)=\inf \left\{1 / r \mid f:\left(D_{r}, i\right) \rightarrow(M, J), f_{*}(0) e=v\right\}, v \in T M .
$$

The above theorem assures $F_{M}$ to be upper semicontinous, implying that

$$
d_{M}(x, y)=\inf \left\{\int \gamma^{*} F_{M} \mid \gamma:[0,1] \rightarrow M, \gamma(0)=x, \gamma(1)=y\right\} .
$$

is well-defined.

Moreover, since an embedded disk can always be perturbed to embedded we prove simultaneously the main properties of the Hanh pseudonorm $S_{M}(v)$, 
which is defined by the same formula as $F_{M}$ with an additional requirement on $f$ to be injective. This pseudometric generates a pseudodistance via path integration, like $F_{M}$ generates $d_{M}$, and this coincides (cf. [K1]) with the distance

$$
h_{M}(x, y)=\inf \sum_{k=1}^{m} d\left(z_{k}, w_{k}\right),
$$

defined via injective chains $f_{k}: D_{1} \rightarrow\left(M^{2 n}, J\right), k=1, \ldots, m, f_{1}\left(z_{1}\right)=p$, $f_{m}\left(w_{m}\right)=q$ and $f_{k}\left(w_{k}\right)=f_{k+1}\left(z_{k+1}\right)$, where $d$ is the Poincare metric on $D_{1}$.

Our approach to the close $\mathrm{PH}$-curve existence result is similar to that of $\underline{\underline{S}}$, where the linearization of the structure $J$ was made at a point. We linearize the structure along the disk and use the reduction of the almost complex problem to a complex one via the Green operator:

$$
T_{r}: C^{k}\left(D_{r}, \mathbb{C}^{n}\right) \rightarrow C^{k+1}\left(D_{r}, \mathbb{C}^{n}\right), \quad g(z) \mapsto \frac{1}{2 \pi i} \iint_{D_{r}} \frac{g(z)}{\zeta-z} d \zeta \wedge d \bar{\zeta} .
$$

It is continuous in the Sobolev and Hölder norms $(\underline{V})$ and obeys the identities: $\bar{\partial} T_{r}=\mathrm{Id},\left.\quad T_{r} \bar{\partial}\right|_{C_{0}^{k+1}}=\mathrm{Id}$.

Proof. We study at first the case, when the curve is embedded. Let $U$ be a neighborhood of the shrunk PH-curve $f_{0}\left(D_{R-\varepsilon}\right)$. We can assume [K1] the disk is standard $f_{0}\left(D_{R-\varepsilon}\right)=D_{R-\varepsilon} \times\{0\}^{n-1} \subset \mathbb{C}^{n}$ and the almost complex structure $J: U \rightarrow \operatorname{End}_{\mathbb{R}}\left(\mathbb{C}^{n}\right), J^{2}=-\mathbf{1}$, along it is the standard complex structure $J(z)=J_{0}$ for all $z \in D_{R-\varepsilon}$. The equation for $f$ to be pseudoholomorphic reads:

$$
\bar{\partial} f+q_{J}(f) \partial f=0, \quad q_{J}(z)=\left[J_{0}+J(z)\right]^{-1} \cdot\left[J_{0}-J(z)\right],
$$

that due to the above properties is equivalent to

$$
\bar{\partial} h=0, \quad h=\left[\operatorname{Id}+T_{R-\varepsilon} \circ q_{J}(f) \circ \partial\right](f) .
$$

For $k \in \mathbb{R} \backslash \mathbb{Z}, k>1$, consider the map

$$
\begin{aligned}
\Phi: \mathcal{J} \times C^{k+1}\left(D_{R-\varepsilon} ; U\right) & \longrightarrow C^{k+1}\left(D_{R-\varepsilon} ; \mathbb{C}^{n}\right), \\
(J, s) & \longmapsto\left[\operatorname{Id}+T_{R-\varepsilon} \circ q_{J}\left(f_{0}+s\right) \circ \partial\right]\left(f_{0}+s\right),
\end{aligned}
$$

where $\mathcal{J}$ is a neighborhood of the given almost complex structure $J$ in $C^{k}$ topology. We consider $U$ as the total space of the "normal bundle", with the sections being denoted by $s$, so that every map $f \in C^{k+1}\left(D_{R-\varepsilon} ; U\right)$, that is $C^{1}$-close to $\left.\left(f_{0}\right)\right|_{D_{R-\varepsilon}}$, has a unique representation $f=f_{0}+s$.

The map $\Phi_{J}=\Phi(J, \cdot)$ is $C^{k}$-smooth and satisfies: $\Phi_{J}(0)=f_{0}, \Phi_{J}^{\prime}(0)=\mathrm{Id}$. It has the Taylor decomposition (with $\|\cdot\|$ being the $C^{k+1}$-norm):

$$
\Phi_{J}(s)=f_{0}+s+o(\|s\|) .
$$

Therefore $\operatorname{Im} \Phi_{J}$ contains a small neighborhood of the curve $f_{0}$.

Let $Z=(a, v) \in T \mathbb{C}^{n}$ and $h_{Z}(z)=a+v z$ be the holomorphic disk in $U$, $z \in D_{R-\varepsilon}$. It is close to $f_{0}$ whenever $Z$ is close to $Z_{0}=(0,(1,0, \ldots, 0)) \in T \mathbb{C}^{n}$. Define

$$
f_{Z}=f_{0}+\Phi_{J}^{-1}\left(h_{Z}\right)
$$


It is a $J$-holomorphic $(R-\varepsilon)$-disk, which satisfies: $f_{Z}-h_{Z}=o\left(\left|Z-Z_{0}\right|\right)$.

Consider the $C^{k}$-map $\Psi: \mathbb{C}^{2 n} \rightarrow \mathbb{C}^{2 n}, Z \mapsto\left(f_{Z}(0),\left(f_{Z}\right)_{*}(0) e\right)$. Since the above estimate implies $\Psi^{\prime}\left(Z_{0}\right)=\mathrm{Id}$, the map $\Psi(Z)$ is a local $C^{k}$-diffeomorphism of a neighborhood of $Z_{0}$. In particular, for every $Z=(a, v)$ sufficiently close to $Z_{0}$ there exists a pair $\tilde{Z}=(\alpha, \zeta)$ such that $\Psi(\tilde{Z})=Z$.

Now the obtained map $f=f_{\tilde{Z}}$ is $C^{1}$-close to $f_{0}$ and so is embedded. It is also smooth due to the usual elliptic regularity ( $\underline{\mathrm{NW}}, \mathbf{M D}, \underline{\mathrm{S}})$ ). If $f_{0}$ is immersed, the reasoning is the same for the neighborhood $U$ obtained via $f_{0}$ by the pull-back.

In the general case for the map $f_{0}:\left(D_{R-\varepsilon}, i\right) \rightarrow(M, J)$ we consider the graph $\hat{f}_{0}:\left(D_{R-\varepsilon}, i\right) \rightarrow\left(D_{R-\varepsilon} \times M, \hat{J}=i \times J\right)$, which is injective and apply the part of the statement already proved.

Remark. The proof implies persistence of big pseudoholomorphic disks (with an insignificant loss of size) under perturbation not only of the initial point and direction, but also of the almost complex structure $J$ (note the role of $\mathcal{J}$ above). This generalizes theorems 1.7 and 3.1.1(ii) in [MD] and [S] respectively.

The properties of the Kobayashi-Royden pseudometric for almost complex manifolds was discussed in [K1. Let us consider the non-integrable version $S_{M}$ of the Hanh pseudometric. It is known $(\mathrm{O})$ that it coincides with the Kobayashi-Royden pseudometric $F_{M}$ for domains $M \subset \mathbb{C}^{n}$ of dimension $n>2$. We generalize this to the non-integrable case.

Theorem 2. $S_{M}=F_{M}$ for almost complex manifolds $\left(M^{2 n}, J\right), n>2$.

Proof. Since $S_{M} \geq F_{M}$, it is enough to show that whatever small $\varepsilon>0$ is, any pseudoholomorphic disk of radius $R>0$ can be approximated by an injective pseudoholomorphic disk of radius $R-\varepsilon$ with the same initial direction.

We give at first a new simple proof of the mentioned above theorem from O. Let $M \subset \mathbb{C}^{n}$ be a domain and $f: D_{R} \rightarrow M$ be a holomorphic map. Denote $f_{W}(z)=f(z)-w_{2} z^{2}-w_{3} z^{3}, z \in D_{R-\varepsilon}, W=\left(w_{2}, w_{3}\right) \in \mathbb{C}^{2 n}$. For small $W$ the map has still the image in $M$. Also note that $f_{W}(0)=f(0)$ and $f_{W}^{\prime}(0)=f^{\prime}(0)$.

By the Sard's theorem a generic $w_{1} \in \mathbb{C}^{n}$ is outside the set

$$
\left\{\frac{f\left(z_{1}\right)-f\left(z_{2}\right)}{z_{1}^{2}-z_{2}^{2}} \mid z_{1}, z_{2} \in D_{R-\varepsilon}\right\} \cup\left\{\frac{f^{\prime}(z)}{2 z} \mid z \in D_{R-\varepsilon}\right\} .
$$

For such a choice the map $f_{w_{2}, 0}$ is injective outside the anti-diagonal $\left\{z_{2}=-z_{1}\right\}$. Note that regularity of the origin is preserved. So, switching on $w_{3}$ being generic, we get the map $f_{w_{2}, w_{3}}$ to be injective everywhere.

In other words, the Sard's theorem implies that the set of $W=\left(w_{2}, w_{3}\right)$ for which $f_{W}$ is not injective has measure zero and so a generic pair of small vectors $w_{2}, w_{3} \in \mathbb{C}^{n}$ defines the required approximating disk $f_{W}(z)$.

In the general complex case we should shift along some holomorphic vector fields. This is achieved by the graph-lift construction and a Royden's lemma $\mathrm{R}$ ] that an embedded holomorphic disk, shrunk a bit, has a Stein neighborhood. 
It is easier, however, to consider the general case of almost complex manifolds $(M, J)$ and to deduce the statement for integrable $J$ as a corollary.

Denote by $\pi: D_{R-\varepsilon} \times M \rightarrow M$ the projection. As in theorem 1, the graphlift $\hat{f}_{0}: D_{R-\varepsilon} \rightarrow D_{R-\varepsilon} \times M$ can be deformed to the family $\hat{f}_{\hat{W}}=\hat{f}_{0}+\hat{\Phi}_{\hat{J}}^{-1}\left(g_{\hat{W}}\right)$, where $g_{\hat{W}}=\hat{w}_{0}+\hat{w}_{1} z-\hat{w}_{2} z^{2}-\hat{w}_{3} z^{3}, \hat{W}=\left(\hat{w}_{2}, \hat{w}_{3}\right), \hat{w}_{j} \in \mathbb{C}^{n+1}$ and $\hat{w}_{0}=$ $\hat{\varphi}_{0}\left(\hat{w}_{2}, \hat{w}_{3}\right), \hat{w}_{1}=\hat{\varphi}_{1}\left(\hat{w}_{2}, \hat{w}_{3}\right)$ are some $C^{k}$-smooth functions, close to zero, such that $\left(\hat{f}_{\hat{W}}^{\delta}(0),\left(\hat{f}_{\hat{W}}^{\delta}\right)_{*}(0) e\right)=\hat{Z}_{0}=(0,(1,0, \ldots, 0)) \in T \mathbb{C}^{n+1}$. We identify above $\hat{f}_{0}$ with $g_{\hat{0}}$, the first coordinate disk, and its neighborhood with a ball $B \subset \mathbb{C}^{n+1}$, equipped with the structure $\hat{J}=i \times J$.

Similarly to the first proof we get: $\hat{f}_{\hat{W}}=g_{\hat{W}}+\rho_{\hat{W}}$, where $\rho_{\hat{W}}=o(|\hat{W}|)$. Now $\hat{f}_{\hat{W}}$ is an embedding if $\pi \hat{f}_{\hat{W}}\left(z_{1}\right) \neq \pi \hat{f}_{\hat{W}}\left(z_{2}\right)$ for $z_{1} \neq z_{2}$ and $\partial \pi \hat{f}_{\hat{W}}(z) \neq 0$. We consider only the first, more complicated, injectivity condition. It's negation is equivalent to $g_{\hat{W}}\left(z_{1}\right)-g_{\hat{W}}\left(z_{2}\right)=\left.\left[\rho_{\hat{W}}\right]\right|_{z_{1}} ^{z_{2}}+\zeta, \zeta \in D$, or after the projection:

$$
w_{2}\left(z_{1}+z_{2}\right)+w_{3}\left(z_{1}^{2}+z_{1} z_{2}+z_{2}^{2}\right)=w_{1}+\frac{\pi \rho_{\hat{W}}\left(z_{2}\right)-\pi \rho_{\hat{W}}\left(z_{1}\right)}{z_{2}-z_{1}}
$$

The last equation is never satisfied for a.e. small $W=\left(w_{2}, w_{3}\right)$ in $\mathbb{C}^{2 n}$. Actually for $w_{1}=\pi \varphi_{1}\left(\hat{w}_{2}, \hat{w}_{3}\right)$ the r.h.s. is $o(|W|)$. Thus the claim follows from the Sard's theorem, if at least one of the coefficients of $w_{2}$ and $w_{3}$ is not small. Since

$$
\begin{aligned}
& D \times D= \\
& =\left[U_{5 \delta}\left(z_{1}=z_{2}=0\right)\right] \cup\left[D \times D \backslash U_{\delta}\left(z_{1}=-z_{2}\right)\right] \cup\left[D \times D \backslash U_{\delta}\left(z_{1}=\left(-\frac{1}{2} \pm \frac{\sqrt{3}}{2}\right) z_{2}\right)\right] .
\end{aligned}
$$

and the regularity at $(0,0)$ is preserved under small perturbation we may achieve injectivity away from the anti-diagonal by the quadratic perturbation and the in its neighborhood by a cubic one. This finishes the proof.

For $n=1$, when almost complex structures are automatically integrable, the equality $S_{M}=F_{M}$ is equivalent to contractibility ( $M$ being a disk). In the case of $\mathbb{C}$-dimension $n=2$ the equality may fail to hold (however arguments of theorem 2 show that $F_{M}$ coincides with the pseudonorm $\tilde{S}_{M}$ obtained via immersed disks).

Example. Consider the map $\varphi_{\alpha}: D_{1} \rightarrow \mathbb{C}^{2}, z \mapsto\left(z(\alpha z-1)^{2}, \alpha z^{2}(\alpha z-1)\right)$, $|\alpha|>1$. It has a unique self-intersection point $\varphi_{\alpha}(0)=\varphi_{\alpha}(1 / \alpha)=0$, which is transversal: $\varphi_{\alpha}^{\prime}(0)=(1,0), \varphi_{\alpha}(1 / \alpha)=(0,1)$, and so non-removable. For a neighborhood $U$ of the image $\operatorname{Im}\left(\varphi_{\alpha}\right)$ the pseudonorms $F_{U}$ and $S_{U}$ are different.

In the product case the pseudonorms $S_{M}$ and $F_{M}$ were compared in [J]. It is however unclear if we can majorize $S_{M} \leq c \cdot F_{M}$, with a constant depending on $M$, or more generally, if Kobayashi and Hanh hyperbolicities ( $d_{M}$ or resp. $S_{M}$ being a metric) are equivalent. Of course, the former implies the latter.

It was shown in $[\mathrm{KO}$ ] that contractible tame almost complex domains are hyperbolic. In other cases the hyperbolicity may be lost. 
Example. Consider the Reeb foliation of $\mathbb{R}^{3}$ with the standard $T^{2}$ as a leaf. This foliation propagates via parallel transports to $\mathbb{R}^{2 n}=\mathbb{R}^{3} \times \mathbb{R}^{2 n-3}, n \geq 2$, and there exists an almost complex structure on $\mathbb{R}^{2 n}$ such that the new foliation is pseudoholomorphic. Every domain containing such a torus is not tame and is not hyperbolic. Note that for $n=2$ only a curve of genus one can be realized as a pseudoholomorphic submanifold in an almost complex $\left(\mathbb{R}^{2 n}, J\right)$ ([M0]). For $n>2$ the sphere $S^{2}$ can be realized as a pseudoholomorphic submanifold, providing similarly a non-tame and non-hyperbolic domain in $\left(\mathbb{R}^{2 n}, J\right)([\mathrm{KO}])$.

Remark. The result of the last theorem shows that the analogy between geodesics and pseudoholomorphic disks, instructive in many respects ([Mo]]), is however limited. Though the Nijenhuis tensor naturally plays the role of the curvature [K2], there are no analogs for the conjugate points in complex time curves theory.

\section{References}

[J] W. Jarnicki, "Kobayashi-Royden vs. Hanh pseudometric in $\mathbb{C}^{2}$, Ann. Polon. Math., 75, no. 3 (2000), 289-294.

[K1] B.S. Kruglikov, "Existence of close pseudoholomorphic disks for almost complex manifolds and application to the Kobayashi-Royden pseudonorm", Funct. Anal. Appl., 33, no.1 (1999), 46-58.

[K2] B.S. Kruglikov, "Tangent and normal bundles in almost complex geometry", prepr. Univ. Troms $\varnothing, 2002-46$.

[KO] B. Kruglikov, M. Overholt, "The Kobayashi pseudodistance on almost complex manifolds", Diff. Geom. Appl., 11 (1999), 265-277.

[MD] D. McDuff, "Singularities of J-holomorphic curves in almost complex 4-manifolds", Journ. Geom. Anal., 2, no. 3 (1992), 249-266.

[Mo] J. Moser, "Pseudo-holomorphic curves on a torus", Proc. Roy. Irish Acad., Sect. A, 95 (1995), suppl., 13-21.

$[\mathrm{NW}]$ A. Nijenhuis, W. Woolf, "Some integration problems in almost-complex and complex manifolds", Ann. Math., 77 (1963), 424-489.

[O] M. Overholt, "Injective hyperbolicity of domains", Ann. Polon. Math., 62, no. 1 (1995), 79-82.

[R] H.L. Royden, "The extension of regular holomorphic maps", Proc. A.M.S., 43, no. 2 (1974), 306-310.

[S] J.-C. Sikorav, "Some properties of holomorphic curves in almost complex manifolds", in "Holomorphic curves in symplectic geometry", M.Audin, J.Lafontaine Eds., Birkhäuser, Progr. in Math., 117 (1994), 165-189.

[V] I. N. Vekua, "Generalized analytic functions" (Russian) Fiz.-Mat. Lit., Moscow (1959); Engl. Transl.: Pergamon, London (1962). 